\title{
Tracking the Kinematically Optimal Trajectories by Mobile Manipulators
}

\author{
Mirosław Galicki ${ }^{1}$ \\ Received: 16 November 2017 / Accepted: 4 May 2018 / Published online: 19 May 2018 \\ (C) The Author(s) 2018
}

\begin{abstract}
This paper addresses the kinematically optimal control problem of the mobile manipulators. Dynamic equations of the mobile manipulator are assumed to be uncertain. Moreover, globally unbounded disturbances are allowed to act on the mobile manipulator when tracking the trajectory by the end-effector. A computationally simple class of the Jacobian transpose control algorithms is proposed for the end-effector trajectory tracking. Such controllers apply a new non-singular Terminal Sliding Mode (TSM) manifold defined by a non-linear integral equality of the second order with respect to the task space tracking error. Based on the Lyapunov stability theory, the proposed Jacobian transpose control schemes are proved to be finite-time stable provided that some well-founded assumptions are fulfilled during the mobile manipulator movement. The performance of the proposed control strategies is illustrated through computer simulations for a mobile manipulator that attains trajectory tracking by the end-effector in a two-dimensional task space and simultaneously minimises some objective function.
\end{abstract}

Keywords Mobile manipulator - Task space trajectory tracking · Kinematically optimal finite-time control · Lyapunov stability

\section{Introduction}

Mobile manipulators have been recently employed to effective practical tasks such as inserting a shaft into a bearing hole or precise parts assembly. As a result of their nature these tasks demand extremely high precision and stability of the performance. In practice one can come across such situations that the tasks mentioned before are specified in terms of a time parameterized geometric path (a trajectory) to be tracked by the end-effector of mobile manipulator. In order to apply known joint space control techniques for tracking such a trajectory, an inverse or pseudo-inverse kinematics algorithm has to be utilised. The process of kinematic inversion is both time consuming (there does not exist, in general, an analytic form of inverse mapping) and becomes very complicated when the Cartesian trajectory generates kinematic and/or algorithmic singularities [15]. Thus, a controller to be designed should accurately track

Mirosław Galicki

m.galicki@ibem.uz.zgora.pl

1 Faculty of Mechanical Engineering, University of Zielona Góra, Zielona Góra, Poland desired end-effector trajectory despite possible singularities met on this trajectory, uncertain dynamic equations, unknown payload to be transferred by the end-effector and external disturbances. Moreover, such controller has to generate at least absolutely continuous control signals (torques) to avoid undesirable chattering. Due to the challenging nature of the aforementioned control design problems, many researchers have proposed different types of controllers. Concerning this context, there are several approaches worth distinguishing. Among them, we mainly focus on the three major ones.

The first approach is the extended or augmented task space formulation (including also input-output linearization techniques) of the inverse kinematics problem showed in works [2-14]. Extending the dimension of the task space by incorporating as many additional constraints as the degree of the redundancy is the essence of it. These additional constraints are obtained based on e.g. various types of optimization criteria. Consequently, the resulting system becomes non-redundant. The control formulations based on the augmented task space technique have some disadvantages. The controllers proposed in [2-11] require inverse of the extended Jacobian matrix that can potentially consist of algorithmic and/or kinematic singularities [15] 
and as a consequence, can produce the control inputs to become unbounded even though the mobile manipulator is not in a singular configuration. Rigorous definitions of singularity for mobile manipulators have been proposed in works [46, 47], which introduce the traditional concept of singularity built on the definition of the extended Jacobian and the concepts of posture and configuration singularity. Although posture and configuration singularity seem to be useful in optimal control problems, they are not suitable in real time control of mobile manipulators. Moreover, the dimensionality of the inverse kinematics problem associated with an extended Jacobian increases. Furthermore, control algorithms from [2-11] require full knowledge of the dynamic equations. The controllers offered in [12-14] need the knowledge of both holonomic end-effector and platform desired trajectories and are not optimal in any sense. Steering signals generated in works $[13,14]$ are discontinuous and require the knowledge of the holonomic manipulator and platform velocities.

The second approach, considered in the works [16-25] is based on the application of the (generalized) pseudoinverse of the mobile manipulator Jacobian matrix in the control formulation. Control algorithms developed from the pseudo-inverse of the Jacobian matrix are attractive and further examined by many researchers, they also have some disadvantages. That is to say, generated controls supply only sub-optimal (and not optimal) solutions. The adaptive control law proposed in [25] needs the knowledge of both holonomic manipulator and platform velocities. The control scheme from [25] involves all the adaptive terms multiplied by the regression matrix that seems to be complex to implement and very time consuming. In the basic monograph [48], the authors have proposed an adaptive robust control of mobile manipulators. Nevertheless, the control laws from [48] require an explicit form of the inverse/pseudo-inverse of the Jacobian matrix as well as desired trajectories of both the end-effector and the mobile platform. Moreover, generated torques/forces belong only to a class of bounded mappings which tend in a limit to discontinuous functions. Furthermore, pseudo-inverse control strategies are not, in general, repeatable (see e.g. $[26,27]$ for stationary robotic manipulators). A concept of repeatability for mobile manipulators was proposed, for the first time, in work [49]. Consequently, an important class of cyclic technological operations (cyclic kinematic tasks) cannot be succeeded in this approach. Furthermore, the application of the pseudo-inverses of the Jacobian matrix is both computationally time consuming and explicitly employs the full rank of this matrix. In order to tackle the singular configurations, the use of damped least-squares has been presented in works [28, 29] instead of the pseudo-inverses. Nonetheless, this technique contains the tracking errors due to a long-term numerical integration drift. Moreover, the controllers mentioned before give only at most asymptotic stability which may be insufficient for completing the tasks that require the extremely high precision.

The third approach proposed in several papers [30-32] is based on the application of the gradient of some potential function. Algorithms from [30-32] solve a regulation problem in a task space. Employing a Filippov solution, works [30-32] may produce discontinuous right hand side of motion equations, which may cause the undesirable effect of chattering.

In recent years, the problem of control design for also nonholonomic mobile robots (mobile platforms) has been the subject of several works. Particularly, in work [52], the authors have shown that more natural optimal motions are related with closed hyperelliptic plane curves with a certain number of loops. The motion planning problem for control-affine systems by using trigonometric polynomials as control functions has been discussed in work [53]. An exhaustive overview of both nonholonomic motion planning and obstacle avoidance has been presented in work [54].

From the literature survey focusing on the mobile manipulator control, one follows that almost all control schemes demand inverse or pseudo-inverse of a Jacobian matrix, what may cause numerical instabilities due to (possible) kinematic and/or algorithmic singularities met on the mobile manipulator trajectory. Moreover, all those algorithms are not able to generate continuous controls resulting in finite-time stability when dynamic equations are uncertain and (unbounded) disturbances act on the mobile manipulator.

This paper presents a significant generalization of the results previously published in works $[35,36,50]$. Namely, works $[35,36,50]$ solve the finite-time control problem in the task (Cartesian) space for only holonomic uncertain dynamic systems, in particular, for stationary robotic manipulators. On the other hand, the present study introduces a new class of controllers being finite-time stable for mobile manipulators (with uncertain dynamics) whose mobile platforms are subject to non-holonomic constraints. In order to eliminate the shortcomings mentioned before, a new task space non-singular Terminal Sliding Mode (TSM) manifold defined by a non-linear integral mapping of the second order with respect to the task space tracking error, is introduced in this paper. The presented TSM manifold enables simultaneously join the first order sliding mode approach possessing the finite-time control capabilities with the second order sliding mode techniques generating the (absolutely) continuous controls. The solution to the trajectory tracking task is based in this work on introducing both a dynamic version of a static computed torque approach presented e.g. in works [33, 34] and useful optimization criterion. By fulfilment of reasonable 
assumption with the respect to the full rank Jacobian matrix, the offered control scheme is shown to be finite-time stable. Furthermore, it supplies at least continuous mappings and therefore avoids the undesirable chattering effect. Our controller consists of a Jacobian transpose matrix multiplied by a non-linear term regarding the task error and its time derivatives, that produces numerical stability even by passing trough singular configurations or near to singular neighbourhoods. The methodology of deriving the control laws in this paper has been taken from the theory of stationary robotic manipulators [35, 36]. Moreover, this study is an essential generalisation of the result given in [37] which only deals with kinematics of the mobile manipulator subject to non-holonomic constraints. On the other hand, the present work analyses both kinematics and uncertain dynamics of the mobile manipulator. In addition, unknown disturbances act on the mobile manipulator when trajectory tracking by the end-effector.

The remainder of the study is organised as follows. Section 2 formulates the finite-time trajectory tracking task. Section 3 establishes a class of kinematically optimal controllers solving the trajectory tracking problem in a finite-time. Section 4 contains computer examples of the end-effector trajectory tracking by a mobile manipulator operating in a two-dimensional task space. Eventually, some closing remarks are pointed out in Section 5. Throughout this paper, $\lambda_{\min }(\cdot), \lambda_{\max }(\cdot)$ denote the minimal and maximal eigenvalues of the matrix $(\cdot)$.

\section{Problem Formulation}

During the operation of the mobile manipulator in the work space, non-holonomic constraints related to the platform motion are induced. They are usually expressed in the so-called Pfaffian form

$A(x) \dot{x}=0$,

where $x \in \mathbb{R}^{l}$ describes the vector of generalized coordinates of the platform; $l \geq 2 ; A(x)$ stands for the $k \times l$ matrix of the full rank (that is, $\operatorname{rank}(A(x))=k$ ) being at least two times differentiable with respect to time; $k$ denotes the number of non-holonomic constraints; $1 \leq k<l$. Let $\operatorname{Ker}(A(x))$ denote a linear sub-space generated by vector fields $a_{1}(x), \ldots, a_{l-k}(x)$. Hence, the kinematic constraint (1) can be correspondingly described by an analytic driftless dynamic system

$\dot{x}=N(x) \alpha$

where $N(x)=\left[a_{1}(x), \ldots, a_{l-k}(x)\right] ; \operatorname{rank}(N(x))=l-k$ and $\alpha=\left(\alpha_{1}, \ldots, \alpha_{l-k}\right)^{T}$ stands for quasi-velocities of the platform (introduced in [1]).
The kinematic equations of the mobile manipulator describe the position and orientation of the end-effector regarding an absolute coordinate system as follows

$p_{e}=f_{e}(q)$,

where $q=\left(x^{T} y^{T}\right)^{T}$ exemplifies the configuration of the mobile manipulator; $y=\left(y_{1}, \ldots, y_{n}\right)^{T} \in \mathbb{R}^{n}$ stands for the joint coordinates of a holonomic manipulator mounted on the platform; $n$ is the number of its kinematic pairs; $p_{e} \in$ $\mathbb{R}^{m}$ denotes the end-effector coordinates; $f_{e}: \mathbb{R}^{l} \times \mathbb{R}^{n} \longrightarrow$ $\mathbb{R}^{m}$ stands for the $m$-dimensional mapping and $m \leq n$ is the dimension of the task space. Differentiating $q$ once and then twice with respect to time and taking into account (2) brings about the following relations:

$\dot{q}=C z, \quad \ddot{q}=C \dot{z}+\dot{C} z$,

where $C=\left[\begin{array}{cc}N(x) & 0 \\ 0 & \mathbb{I}_{n}\end{array}\right] ; z=\left(\begin{array}{c}\alpha \\ \dot{y}\end{array}\right) \in \mathbb{R}^{l+n-k}$ is the vector of reduced velocities; $\mathbb{I}_{n}$ denotes $n \times n$ identity matrix.

The control scheme designed in the next section is applicable to non-holonomic mechanical systems comprising mobile manipulators considered here. The dynamics of a mobile manipulator described in reduced coordinates is given by the following equations [21-24]:

$M(q) \dot{z}+P(q, z) z+G(q)+D(t, q, z)=B v$,

where $M$ denotes the $(l+n-k) \times(l+n-k)$ symmetric positive definite reduced inertia matrix; $P$ is the $(l+$ $n-k$ )-dimensional reduced vector representing centrifugal and Coriolis forces; $G$ stands for the mobile manipulator reduced gravity forces; $D$ means the $(l+n-k)$-dimensional external disturbance signal whose time-derivative $\dot{D}$ is (by assumption) a locally bounded Lebesgue measurable mapping; $B$ denotes the $(l+n-k) \times(l+n-k)$ matrix describing the configuration variables of the platform and holonomic manipulator which are directly driven by the actuators and $v$ represents the $(l+n-k)$-dimensional vector of controls (torques/forces). Without loss of generality, $\|D\|$ and $\|\dot{D}\|$ are assumed to be upper estimated as follows

$\|D\| \leq \alpha_{0}(t, q, z), \quad\|\dot{D}\| \leq \alpha_{1}(t, q, z)$,

where $\alpha_{0}$ and $\alpha_{1}$ stand for the time dependent known non-negative and locally bounded Lebesgue measurable functions, respectively.

The consequence of inequality $l+n>m+k$ (one can observe that mobile manipulator considered herein is a redundant mechanism) is the possibility to augment the end-effector conventional trajectory tracking (primary task) with additional user-specified useful task coordinates $p_{a} \in$ $\mathbb{R}^{l+n-m-k}$ (secondary task) of the following general form:

$p_{a}=f_{a}(q)$,

where $f_{a}: \mathbb{R}^{l+n} \longrightarrow \mathbb{R}^{l+n-m-k}$ stands for a given at least triply differentiable mapping with respect to $q$. It is 
practically desirable to generate joint trajectory $q=q(t)$ in such a way as to minimize an objective (kinematic) function $\mathcal{F}(q)$. Based on the kinematic criterion $\mathcal{F}(q)$, which is assumed to be at least four times differentiable with respect to $q$, the general form for $f_{a}$, proposed, e.g., in works [11, $15]$ may be expressed as

$f_{a}=\mathcal{N}(q) \frac{\partial \mathcal{F}(q)}{\partial q}$,

where $\mathcal{N}$ stands for the $(l+n-m-k) \times(l+n)$ orthogonal

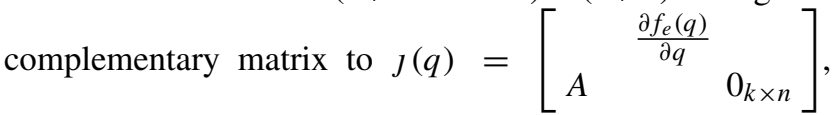
i.e., $J \mathcal{N}^{T}=0,0_{k \times n}$ is the $k \times n$ zero matrix. Let us note that $J(q)$ denotes an auxiliary Jacobian matrix which is related to necessary condition of minimum of objective function $\mathcal{F}$ subject to both holonomic constraints (3) and non-holonomic ones (1) (see [11] for details). Consequently, $\mathcal{N}(q) \frac{\partial \mathcal{F}(q)}{\partial q}=0$ present $n+l-m-k$ transversality conditions which together with Eqs. 3 and 1 make it possible to uniquely determine optimal mobile manipulator configuration $q$ corresponding to a fixed endeffector location $p_{e}$

In an further analysis, we shall employ a simple and practically useful optimization criterion for redundancy resolution with a cost function

$\mathcal{F}(q)=\frac{c_{\mathcal{F}}}{2}\left\langle q-q_{\text {rest }}, K_{\mathcal{F}}\left(q-q_{\text {rest }}\right)\right\rangle$,

where $q_{\text {rest }}$ is some rest (preferred) posture; $\langle$,$\rangle denotes$ the scalar product of vectors; $c_{\mathcal{F}}$ is a positive constant; $K_{\mathcal{F}}$ stands for a positive definite diagonal weighting matrix. Let us notice that for $q_{\text {rest }}=q(0)$, minimization of Eq. 9 avoids sudden changes of configurations and enables the mobile manipulator to reduce the values of controls in $L_{2}$ norm by appropriate weighting of joints closer to the mobile platform as the computer simulations given in Section 4 will show. By concatenating $f_{e}(q)$ with $f_{a}(q)$, one obtains the general kinematic and differential mappings between $q$ and extended task coordinates $p=\left(p_{e}^{T} p_{a}^{T}\right)^{T}$

$p=f(q), \quad \dot{p}=J z$,

where $f=\left(f_{e}^{T} f_{a}^{T}\right)^{T}$ and $J=\frac{\partial f}{\partial q} C$ is the $(l+n-k) \times$ $(l+n-k)$ extended Jacobian matrix. Let us also observe that the equality on the left hand side of Eq. 10 for a fixed $p=\left(\begin{array}{c}p_{e} \\ 0\end{array}\right)$ together with Eq. 1 result in $n+l$ non-linear algebraic/differential equations to determine optimal mobile manipulator configuration $q \in \mathbb{R}^{n+l}$.

The task of the mobile manipulator is to track both a desired end-effector trajectory $p_{d}^{e}(t) \in \mathbb{R}^{m}, t \in[0, \infty)$ with $p_{d}^{e}(\cdot) \in C^{3}[0, \infty)$ and desired user specified trajectory $p_{d}^{a}(t) \in \mathbb{R}^{l+n-m-k}$ that equals $p_{d}^{a}(t)=0$ for $f_{a}$ given by Eq. 8 . By employing the task tracking error $e=\left(\left(e^{e}\right)^{T}\left(e^{a}\right)^{T}\right)^{T}=f(q)-p_{d}(t)$, where $p_{d}=$
$\left(\left(p_{d}^{e}\right)^{T}\left(p_{d}^{a}\right)^{T}\right)^{T} ; e^{e}=f_{e}-p_{d}^{e} ; e^{a}=f_{a}-p_{d}^{a}$, the task space finite-time control problem may be formally expressed by means of the following equations:

$\lim _{t \rightarrow T} e(t)=0, \lim _{t \rightarrow T} \dot{e}(t)=0, \lim _{t \rightarrow T} \ddot{e}(t)=0$,

where $0 \leq T$ denotes a finite-time of convergence of $f(q)$ to $p_{d}$ and $e(t)=\dot{e}(t)=\ddot{e}(t)=0$ for $t \geq T$. Let us notice that the left-hand side equation of Eq. 11 presents for $t \geq T$ and $f_{a}=\mathcal{N}(q) \frac{\partial \mathcal{F}(q)}{\partial q}$ a necessary condition for relative minimum of $\mathcal{F}$. For the purpose of the further analysis, $J$ is expected to be of the full rank in the operation region of the end-effector, i.e.,

$\operatorname{rank}(J(q))=l+n-k$.

Based on Eqs. 4-5, our aim is to obtain at least absolutely continuous vector function of controls $v \in \mathbb{R}^{l+n-k}$ such that $\dot{q}=C z$

$\dot{z}=M^{-1} B v-M^{-1}(P z+G+D)$

and joint trajectory $q=q(t)$ corresponding to the solution of differential equations (13), fulfils relations (11). In the further analysis, useful properties of both dynamic equations (5) and kinematic mapping (10) are summarised that will be applied while designing the controller. In the case of revolute kinematic pairs of the holonomic manipulator, the following inequalities hold true:

$$
\begin{aligned}
& \|B\|_{F} \leq w_{B},\|P z\| \leq w_{P}\|z\|^{2},\|G\| \leq w_{G} \\
& \|J\|_{F},\left\|\frac{\partial J}{\partial q}\right\|_{F},\left\|\frac{\partial^{2} J}{\partial q^{2}}\right\|_{F} \leq w_{1}+w_{2}\left\|q-q_{\text {rest }}\right\|,
\end{aligned}
$$

where $\|\cdot\|_{F}$ is the Frobenius (Euclidean) norm of the matrix $(\cdot) ; w_{B}, w_{P}, w_{G}, w_{1}, w_{2}$ are known positive scalar coefficients. Moreover, from Eq. 12 and the property of inertia matrix $M$ in Eq. 5, one obtains that

$0<\lambda_{\min }\left(J M^{-1} J^{T}\right) \mathbb{I}_{l+n-k} \leq J M^{-1} J^{T}$,

where $\mathbb{I}_{l+n-k}$ denotes the $(l+n-k) \times(l+n-k)$ identity matrix. Our aim is to obtain at least absolutely continuous control $v$. For this purpose, let us differentiate dynamic equations (5) with respect to time

$M \ddot{z}=B \dot{v}+\dot{B} v-\dot{M} \dot{z}-\frac{d}{d t}(P z+G+D)$.

Hence

$\ddot{z}=M^{-1} B \dot{v}+\mathcal{R}(t, q, z, t)$,

where $\mathcal{R}=M^{-1}\left(\dot{B} v-\dot{M} \dot{z}-\frac{d}{d t}(P z+G+D)\right)$. Partly inspired by the control methodology borrowed from the stationary robotic manipulators $[35,36]$, now we propose a dynamically computed torque vector $\dot{v}$ of the form

$\dot{v}=B^{-1} J^{T} u$,

where $u \in \mathbb{R}^{l+n-k}$ is a new control to be determined. Let us note that for the non-holonomic platforms of type $(2,0)$, considered herein - see Fig. 1, the actuation matrix $B$ in 
Eq. 5 is diagonal with positive elements. Consequently, it is invertible.

Replacing $\dot{v}$ in Eq. 17 by the right-hand side of Eq. 18 gives the expression dependent on the extended Jacobian matrix $J$

$\ddot{z}=M^{-1} J^{T} u+\mathcal{R}$.

Let $a$ be a real positive number which fulfils the following inequality:

$0<a \leq \lambda_{\min }\left(J M^{-1} J^{T}\right)$.

The aim of the further considerations is to find input signal $u(t)$ and the corresponding control vector $v(t)$ by solving the differential equations (18), such that augmented endeffector location vector $p$ follows $p_{d}$. Therefore, let us triply differentiate $e$ with respect to time thus obtaining

$\frac{d^{3} e}{d t^{3}}=J \ddot{z}+\dot{J} \dot{z}+\frac{d}{d t}\left(\dot{J}_{z}\right)-\frac{d^{3} p_{d}}{d t^{3}}$.

Let us also note that it is easy to present the system of differential equations (13), (19) and (21) in a normal form. For this purpose, $\ddot{z}$ in the right-hand side of Eq. 21 is replaced by the right-hand side of Eq. 19 thus obtaining

$$
\begin{aligned}
\frac{d^{3} e}{d t^{3}}= & J M^{-1} B \dot{v}+J \mathcal{R}+\dot{J}\left(M^{-1} B v-M^{-1}(P z+G+D)\right) \\
& +\frac{d}{d t}\left(\dot{J}_{z}\right)-\frac{d^{3} p_{d}}{d t^{3}} .
\end{aligned}
$$

Consequently, we obtain a normal system of differential equations (with explicitly written higher order derivatives) whose right-hand sides depend on $t, q, z, v$ and a new control $\dot{v}$ to be determined. Using the error dynamics (22), an approach, applying the Lyapunov stability theory to the solution of the control problem (11), (13) and (22), will be shown in the next section.

\section{Kinematically Optimal Control for the Mobile Manipulator}

In order to design our controllers and then show their properties, some useful sliding vector will now be introduced which overcomes the limitations and shortcomings of the first order classic sliding variables expressed in joint coordinates [38-40]. For this aim, let $s=\left(s_{1}, \ldots, s_{l+n-k}\right)^{T} \in$ $\mathbb{R}^{l+n-k}$ be a task space sliding vector variable. The following non-singular terminal sliding manifold is proposed:

$$
\begin{aligned}
\mathcal{S}= & \{(s(t), \ddot{e}(t), \ddot{e}, \dot{e}, e): s(t)=\ddot{e}(t) \\
& \left.+\int_{0}^{t}\left(\lambda_{2} \ddot{e}^{3 / 5}+\lambda_{2} \lambda_{1}^{3 / 5}\left(\dot{e}^{9 / 7}+\lambda_{0}^{9 / 7} e\right)^{1 / 3}\right) d \tau\right\},
\end{aligned}
$$

where $s(t), \ddot{e}(t) \in \mathbb{R}^{n+l-k} ; \ddot{e} \in A C\left([0, \infty), \mathbb{R}^{n+l-k}\right)$; $A C(\cdot)$ denotes a class of absolutely continuous functions; $\dot{e} \in C^{1}\left([0, \infty), \mathbb{R}^{n+l-k}\right) ; e \in$ $C^{2}\left([0, \infty), \mathbb{R}^{n+l-k}\right) ; \lambda_{0}=\operatorname{diag}\left(\lambda_{0,1}, \ldots, \lambda_{0, l+n-k}\right) ;$ $\lambda_{1}=\operatorname{diag}\left(\lambda_{1,1}, \ldots, \lambda_{1, l+n-k}\right) ; \lambda_{2}=$

$\operatorname{diag}\left(\lambda_{2,1}, \ldots, \lambda_{2, l+n-k}\right) ; \lambda_{i, j}$ denote positive coefficients (controller gains); $i=0: 2 ; j=1: l+n-k$. The potency of both $e, \dot{e}, \ddot{e}$ and $\lambda_{0}, \lambda_{1}, \lambda_{2}$ is defined componentwise. From the definition of $\mathcal{S}$ in Eq. 23, it follows that $\operatorname{dim}(\mathcal{S})=\infty$. Moreover, $\operatorname{codim}(\mathcal{S})=n+l-k$. Set $\mathcal{S} \subset$ $\mathbb{R}^{n+l-k} \times \mathbb{R}^{n+l-k} \times A C \times C^{1} \times C^{2}$ is also called an embedded manifold in $\mathbb{R}^{n+l-k} \times A C \times C^{1} \times C^{2}$. Let us note that equality $\ddot{e}(t)+\int_{0}^{t}\left(\lambda_{2} \ddot{e}^{3 / 5}+\lambda_{2} \lambda_{1}^{3 / 5}\left(\dot{e}^{9 / 7}+\lambda_{0}^{9 / 7} e\right)^{1 / 3}\right) d \tau=0$ is equivalent to a known homogeneous triple integral system of negative degree equal to $-\frac{2}{9}$. The finite-time stability of such systems was proved e.g. in [51]. The exponents in Eq. 23 ensure the finite-time convergence to the origin one. Differentiating $s$ in Eq. 23 with respect to time and then replacing $\frac{d^{3} e}{d t^{3}}$ by the right-hand side of Eq. 21, one obtains

$\dot{s}=J \ddot{z}+\mathcal{W}(q, z, v, t, e, \dot{e}, \ddot{e})$

where $\mathcal{W}=\lambda_{2} \ddot{e}^{3 / 5}+\lambda_{2} \lambda_{1}^{3 / 5}\left(\dot{e}^{9 / 7}+\lambda_{0}^{9 / 7} e\right)^{1 / 3}-\frac{d^{3} p_{d}}{d t^{3}}+\dot{J} \dot{z}+$ $\frac{d}{d t}(\dot{J} z)$.

In the subsequent analysis, we shall need an upper estimate of the Euclidean norm of expression $J \mathcal{R}+\mathcal{W}$. Basing on Eqs. 4, 13, 17 and 14, we find an upper estimate on $\|J \mathcal{R}+\mathcal{W}\|$ which takes the form

$\|J \mathcal{R}+\mathcal{W}\| \leq \mathcal{U}(q, z, v, t, e, \dot{e}, \ddot{e})$,

where $\mathcal{U}=\left(w_{1}+w_{2}\left\|q-q_{\text {rest }}\right\|\right)\left(w_{3}\|v\|\|z\|+w_{4}\|z\|^{3}+\right.$ $\left.w_{5}\left(\|z\|+\|z\| \alpha_{0}\right)+w_{6} \alpha_{1}\right)+\| \lambda_{2} \ddot{e}^{3 / 5}+\lambda_{2} \lambda_{1}^{3 / 5}\left(\dot{e}^{9 / 7}+\right.$ $\left.\lambda_{0}^{9 / 7} e\right)^{1 / 3}-\frac{d^{3} p_{d}}{d t^{3}} \| ; w_{3}, \ldots, w_{6}$ are known positive scalar coefficients (construction parameters of the mobile manipulator). In the further study, we need a useful result [36].

Lemma 1 If $s=0$ then task errors $(e, \dot{e}, \ddot{e})$ converge in a finite-time to the origin $(e, \dot{e}, \ddot{e})=(0,0,0)$.

We propose now a (simple) kinematically optimal control law of the form given below which fulfils equality constraints (11)

$u(t, q, z, v, e, \dot{e}, \ddot{e}, s)= \begin{cases}-\frac{c}{a} \frac{s}{\|s\|}\left(\mathcal{U}+c_{0}\right) & \text { for } s \neq 0 \\ 0 & \text { otherwise }\end{cases}$

where $\dot{e}=J z-\dot{p_{d}} ; \ddot{e}=J \dot{z}+\dot{J}_{z}-\ddot{p}_{d} ; c, c_{0}$ are positive constant gains to be specified further on. Based on Eqs. 18 and 26, we can find an absolutely continuous control vector $v$ by solving (in the Filippov sense [41]) the following differential equations:

$\dot{v}=B^{-1} J^{T} u(t, q, z, v, e, \dot{e}, \ddot{e}, s)$. 
The existence of the solution of differential equations (27) has been shown in our recent work [50]. Since, the righthand side of Eq. 27 is a non-Lipschitz and complex mapping (in fact, it is discontinuous), we assume in further analysis that the solution of closed-loop system (5), (27) is unique.

The aim of the further examination is to provide conditions on controller gains $\lambda_{0}, \lambda_{1}, \lambda_{2}, c$ and $c_{0}$, which guarantee the fulfilment of equalities (11). Before we present our main results, the rigorous definition of stable convergence in a finite time is given below.

Definition 1 The origin $(e, \dot{e}, \ddot{e})=(0,0,0)$ is said to be stable convergent in a finite time if it is Lyapunov stable and the solution of differential equations (22), (27) and (13), which starts from $(e(0), \dot{e}(0), \ddot{e}(0))$ at time $t=0$ attains the origin in a finite time $T(e(0), \dot{e}(0), \ddot{e}(0))<$ $\infty$, i.e., $\lim _{t \rightarrow T}(e(t), \dot{e}(t), \ddot{e}(t))=\left(\begin{array}{lll}0, & 0, & 0\end{array}\right)$ and $\lim _{(e, \dot{e}, \ddot{e}) \rightarrow(0,0,0)} T=0$.

Based on the application of the Lyapunov stability theory, we are now ready to give the following result.

Theorem 1 If $J$ fulfils inequality (20) along desired trajectory $p_{d}$ and $\lambda_{0}, \lambda_{1}, \lambda_{2}, c_{0}>0, c \geq 1$ then control scheme (13), (26), (27) enables stable convergence in a finite time of the task space tracking errors $(e, \dot{e}, \ddot{e})$ to the $\operatorname{origin}(e, \dot{e}, \ddot{e})=(0,0,0)$.

Proof Let us consider the following Lyapunov function candidate:

$V=\frac{1}{2}\langle s, s\rangle$.

Computing the time derivative of Eq. 28 along the trajectories of Eq. 24 results in the following expression:

$\dot{V}=\langle s, J \ddot{z}+\mathcal{W}\rangle$.

On account of Eqs. 17 and 29, we have

$\dot{V}=\left\langle s, J M^{-1} B \dot{v}\right\rangle+\langle s, J \mathcal{R}+\mathcal{W}\rangle$.

Inserting the right hand side of Eq. 27 into 30, one obtains

$\dot{V}=\left\langle s, J M^{-1} J^{T} u\right\rangle+\langle s, J \mathcal{R}+\mathcal{W}\rangle$.

Replacing $u$ in Eq. 31 by the right hand side of Eq. 26 results in

$\dot{V}=-\left\langle s, J M^{-1} J^{T} \frac{c}{a} \frac{s}{\|s\|}\left(\mathcal{U}+c_{0}\right)\right\rangle+\langle s, J \mathcal{R}+\mathcal{W}\rangle$.

Basing on Eqs. 15 and 20, we get

$\dot{V} \leq-c\|s\|\left(\mathcal{U}+c_{0}\right)+\langle s, J \mathcal{R}+\mathcal{W}\rangle$.

The next step of the proof is to estimate the scalar product in Eq. 33. On account of inequality (25), we have that

$\langle s, J \mathcal{R}+\mathcal{W}\rangle \quad \leq\|s\| \mathcal{U}$.
Therefore, basing on the assumption $c \geq 1$ from Theorem 1 , one easily gets that

$\dot{V} \leq-c\|s\|\left(\mathcal{U}+c_{0}\right)+\|s\| \mathcal{U} \leq-c c_{0}\|s\|$.

Let us notice that $c c_{0}>0$. Hence, inequality (35) proves that $\operatorname{TSM} s=0$ is attainable in a finite time less or equal to $\frac{\sqrt{2 V(0)}}{c c_{0}}$. Finally, from Lemma 1, one follows that the origin $(e, \dot{e}, \ddot{e})=(0,0,0)$ can be attained in a finite time $T$.

We can make a few remarks with respect to the control law (26), (27) and Theorem 1.

- Remark 1. If the task of the control is only to track desired end-effector trajectory $p_{d}^{e}$ (without objective function $\mathcal{F}$ ), then mobile manipulator becomes strictly redundant, i.e., $l+n>m$. If this is the case, we can define the task space TSM $s^{e}$ as $s^{e}=$ $\ddot{e}^{e}+\int_{0}^{t}\left(\lambda_{2, e}\left(\ddot{e}^{e}\right)^{3 / 5}+\lambda_{2, e} \lambda_{1, e}^{3 / 5}\left(\left(\dot{e}^{e}\right)^{9 / 7}+\lambda_{0, e}^{9 / 7} e^{e}\right)^{1 / 3}\right) d \tau$, where $\lambda_{0, e}, \lambda_{1, e}, \lambda_{2, e}$ stand for the controller gains. Thus, control law (26)-(27) may be expressed in the following form:

$\dot{v}^{e}=B^{-1}\left(j^{e}\right)^{T} u^{e}\left(t, q, z^{e}, v^{e}, e^{e}, \dot{e}^{e}, \ddot{e}^{e}, s_{e}\right)$.

where $j^{e}=\frac{\partial f_{e}}{\partial q} C$ and

$u^{e}= \begin{cases}-\frac{c_{e}}{a_{e}} \frac{s^{e}}{\left\|s^{e}\right\|}\left(\mathcal{U}_{e}+c_{0}^{e}\right) & \text { for } s^{e} \neq 0 \\ 0 & \text { otherwise },\end{cases}$

where $\mathcal{U}_{e}=\| \lambda_{2, e}\left(\ddot{e}^{e}\right)^{3 / 5}+\lambda_{2, e} \lambda_{1, e}^{3 / 5}\left(\left(\dot{e}^{e}\right)^{9 / 7}+\lambda_{0, e}^{9 / 7}\right.$ $\left.e^{e}\right)^{1 / 3}-\frac{d^{3} p_{d}^{e}}{d t^{3}}\left\|+w_{3}\right\| v\|\| z\left\|+w_{4}\right\| z \|^{3}+w_{5}(\|z\|+$ $\left.\|z\| \alpha_{0}\right)+w_{6} \alpha_{1} ; c_{e}>1, c_{0}^{e}$ are positive constant gains; $a_{e}$ stands for the estimation of the minimal eigenvalue of matrix $j^{e} M^{-1}\left(j^{e}\right)^{T}$.

- Remark 2. If $J(\cdot)$ is singular at configuration $q^{\prime}=q\left(t^{\prime}\right)$ for some time instant $t^{\prime}$ and $0 \neq s \notin \operatorname{ker}\left(J^{T}\left(q^{\prime}\right)\right)$ then for sufficiently large $c$, term $-c\left\langle s, J M^{-1} J^{T} s\right\rangle$ in Eq. 32 can take arbitrarily large negative values which implies negative value of time derivative $\dot{V}$. Consequently, controller (26)-(27) is able to generate mobile manipulator trajectory $q=q(t)$ which can transversally pass through singular manifold $\{q$ : $\operatorname{det}(J(q))=0\}$ at configuration $q^{\prime}=q\left(t^{\prime}\right)$.

- Remark 3. Let us notice that for a special form of auxiliary kinematic function $f_{a}$ equal to

$f_{a}(q)=W q$,

where $W$ stands for a given $(l+n-m-k) \times$ $(l+n)$ constant matrix, the Frobenius norm of the Jacobian $\frac{\partial}{\partial q}\left(\left(f_{e}(q)\right)^{T},(W q)^{T}\right)^{T}$ fulfils inequality (14). Consequently, an auxiliary function (38) provides the same controller as that given by expressions (26)(27) with $e=\left(\begin{array}{l}f_{e}(q)-p_{d}^{e}(t) \\ W q-p_{d}^{a}(t)\end{array}\right)$. 
Let us observe that controller (26) depends on construction parameters (constants) $w_{1}, \ldots, w_{6}$ whose accurate determination is not an easy task in practice. In the subsequent analysis, we shall try both to reduce the number of essential construction parameters and to apply only their corresponding estimates by designing the controller. For this purpose, we use the following auxiliary inequality:

$\mathcal{U} \leq \gamma \cdot \mathcal{X}(q, z, v, t, e, \dot{e}, \ddot{e})$,

where $\gamma=\max \left\{\max \left\{w_{1}, w_{2}\right\} \cdot \max \left\{w_{3}, w_{4}, w_{5}, w_{6}\right\}, 1\right\}$; $\mathcal{X}=\left(1+\| q-q_{\text {rest }}||\right) \cdot\left(\|v\|\||| z\|+\|z\|^{3}+\|z\|\left(1+\alpha_{0}\right)+\right.$ $\left.\alpha_{1}+\left\|\lambda_{2} \ddot{e}^{3 / 5}+\lambda_{2} \lambda_{1}^{3 / 5}\left(\dot{e}^{9 / 7}+\lambda_{0}^{9 / 7} e\right)^{1 / 3}-\frac{d^{3} p_{d}}{d t^{3}}\right\|\right)$. As is easy to see, controller

$u(t, q, z, v, e, \dot{e}, \ddot{e}, s)= \begin{cases}-\frac{c}{a} \frac{s}{\|s\|}\left(\gamma \mathcal{X}+c_{0}\right) & \text { for } s \neq 0 \\ 0 & \text { otherwise }\end{cases}$

guarantees a stable convergence in a finite time of the task errors $(e, \dot{e}, \ddot{e})$ to the origin $(e, \dot{e}, \ddot{e})=(0,0,0)$. Let $\hat{\gamma}$ be an estimate of the unknown construction parameter $\gamma$. In the sequel, estimate $\hat{\gamma} \geq 1$ is assumed to fulfil the following inequality:

$|\hat{\gamma}-\gamma| \leq \delta$

where $\delta$ denotes a given accuracy of the estimation. Let us notice that, in fact, $\delta$ is not subjected to any limitations. Based on Eq. 41, we propose kinematically optimal control law which is convenient from the practical point of view and given by the following expression:

$u(t, q, z, v, e, \dot{e}, \ddot{e}, s)=\left\{\begin{array}{ll}-\frac{c}{a} \frac{s}{\|s\|}\left(\hat{\gamma} \mathcal{X}+\delta \mathcal{X}+c_{0}\right) & \text { for } s \neq 0 \\ 0 & \text { otherwise }\end{array}\right.$.

We are now in position to give the following theorem.

Theorem 2 If J fulfils the inequality (20) along the desired trajectory $p_{d}$ and $\lambda_{0}, \lambda_{1}, \lambda_{2}, c_{0}>0, \hat{\gamma}$ satisfies (41), $c \geq 1$ then control scheme (13), (27), (42) guarantees stable convergence in a finite time of the task space tracking errors $(e, \dot{e}, \ddot{e})$ to the origin $(e, \dot{e}, \ddot{e})=(0,0,0)$.

Proof Consider the following Lyapunov function candidate:

$V=\frac{1}{2}\langle s, s\rangle$.

Differentiating (43) with respect to time and taking into account (17)-(18) and (24), one obtains that

$\dot{V}=\left\langle s, J M^{-1} J^{T} u+J \mathcal{R}+\mathcal{W}\right\rangle$.

Let us insert the right-hand side of (42) into (44). After simple calculations and use of both inequality (39) and assumption $c \geq 1$ from Theorem 2, we obtain the following upper estimate on the time-derivative of $V$ :

$\dot{V} \leq c\|s\| \gamma \mathcal{X}-\left\langle s, J M^{-1} J^{T} \frac{c}{a} \frac{s}{\|s\|}\left(\hat{\gamma} \mathcal{X}+\delta \mathcal{X}+c_{0}\right)\right\rangle$.

On account of Eqs. 15 and 20, we get

$\dot{V} \leq c\|s\| \gamma \mathcal{X}-c\|s\|\left(\hat{\gamma} \mathcal{X}+\delta \mathcal{X}+c_{0}\right)$.

Finally, the upper estimate on $\dot{V}$ takes the form given below

$\dot{V} \leq c\|\mid s\| \mathcal{X}(\gamma-\hat{\gamma}-\delta)-c c_{0}\|s\|$.

Since $\gamma-\hat{\gamma}-\delta<0$ and $c c_{0}>0$, inequality (47) proves that TSM $s=0$ is attainable in a finite time less or equal to $\frac{\sqrt{2 V(0)}}{c c_{0}}$. Consequently, Lemma 1 implies that the origin $(e, \dot{e}, \ddot{e})=(0,0,0)$ is attainable in a finite time $T$.

Let us note that all of the controllers (26), (37), (40), (42) require the knowledge of quantities $q, z=\left(\begin{array}{c}\alpha \\ \dot{y}\end{array}\right)$, $e, \dot{e}$ and $\ddot{e}$, respectively to generate suitable torques/forces. In most cases, real mobile manipulators are equipped with encoders which measure angular displacements of the wheels of the platform and angles in the kinematic pairs of the holonomic manipulator. Moreover, many commercial sensors are available for measurement of the end-effector position $p_{e}$, such as vision systems, electromagnetic measurement systems, position sensitive detectors or laser tracking systems. Consequently, task error $e$ appearing in the proposed controllers is also assumed to be available from measurements. Hence, reconstruction of auxiliary velocity $\alpha$, joint velocity $\dot{y}$ of the holonomic manipulator, task error velocity $\dot{e}$ and task error acceleration $\ddot{e}$ is required to apply controllers (26), (37), (40), (42) in practice. Let us note that for the mobile platforms belonging to a class $(2,0)$, auxiliary velocities $\alpha$ are equal to scaled angular velocities $\dot{\phi}=\left(\begin{array}{c}\dot{\phi}_{1} \\ \dot{\phi}_{2}\end{array}\right)$ of the platform wheels (see Fig. 1), i.e., $\alpha=\frac{R}{2} \dot{\phi}$, where $R$ denotes the radius of the wheel. As a result, $z=\frac{d}{d t}\left(\left[\begin{array}{cc}\frac{R}{2} \mathbb{I}_{2} & 0 \\ 0 & \mathbb{I}_{n}\end{array}\right]\left(\begin{array}{l}\phi \\ y\end{array}\right)\right)$ and its reconstruction is equivalent to reconstruction of $\dot{\phi}$ and $\dot{y}$, respectively ( $\phi$ and $y$ are available from mobile manipulator encoders). There exist many approaches in the literature to reconstruct quantities $z, \dot{e}$ and $\ddot{e}$, respectively (see e.g. our recent works $[36,50]$, in which different kinds of state observers were analysed) A computationally efficient approach has been recently proposed in works [42, 43] to numerically find derivatives of absolutely continuous functions. On account of the fact that angles $\phi, y$ and task error $e=$ $e(t)$ are known (measurable), one can exactly reconstruct reduced velocity $z(t)$, task error velocity $\dot{e}(t)$ and task error acceleration $\ddot{e}(t)$ (by neglecting the measurement noise 
of a device) after finite-times of transient processes, say $T_{z}^{\prime}, T_{e}^{\prime}>0$, respectively. The first-order uniform robust exact differentiator (model-free observer) for reconstruction of $z$ and the second-order uniform robust exact differentiator reconstructing $\dot{e}$ and $\ddot{e}$, respectively, take in our case the following forms:

$$
\begin{aligned}
\dot{z}_{0}= & z_{1}-\hat{\lambda}_{1}^{z} L_{z}(t)^{1 / 2}\left|z_{0}-\left[\begin{array}{cc}
\frac{R}{2} \mathbb{I}_{2} & 0 \\
0 & \mathbb{I}_{n}
\end{array}\right]\left(\begin{array}{l}
\phi \\
y
\end{array}\right)\right|^{1 / 2} \\
& \operatorname{sign}\left(z_{0}-\left[\begin{array}{cc}
\frac{R}{2} \mathbb{I}_{2} & 0 \\
0 & \mathbb{I}_{n}
\end{array}\right]\left(\begin{array}{c}
\phi \\
y
\end{array}\right)\right) \\
\dot{z}_{1}= & -\hat{\lambda}_{0}^{z} L_{z}(t) \operatorname{sign}\left(z_{0}-\left[\begin{array}{cc}
\frac{R}{2} \mathbb{I}_{2} & 0 \\
0 & \mathbb{I}_{n}
\end{array}\right]\left(\begin{array}{l}
\phi \\
y
\end{array}\right)\right),
\end{aligned}
$$

and

$$
\begin{aligned}
& \dot{\eta}_{0}=\eta_{1}-\hat{\lambda}_{2}^{e} L_{e}(t)^{1 / 3}\left|\eta_{0}-e\right|^{2 / 3} \operatorname{sign}\left(\eta_{0}-e\right), \\
& \dot{\eta}_{1}=\eta_{2}-\hat{\lambda}_{1}^{e} L_{e}(t)^{2 / 3}\left|\eta_{0}-e\right|^{1 / 3} \operatorname{sign}\left(\eta_{0}-e\right), \\
& \dot{\eta}_{2}=-\hat{\lambda}_{0}^{e} L_{e}(t) \operatorname{sign}\left(\eta_{0}-e\right),
\end{aligned}
$$

where $\hat{\lambda}_{0}^{z}, \hat{\lambda}_{1}^{z}, \hat{\lambda}_{0}^{e}, \hat{\lambda}_{1}^{e}, \hat{\lambda}_{2}^{e}$ are positive constants whose values were suggested in [42, 43]; $z_{1}, \eta_{1}, \eta_{2}$ denote the outputs of differentiators (48)-(49) reconstructing exactly reduced velocity $z(t)$, task error velocity $\dot{e}(t)$ and task error acceleration $\ddot{e}(t)$, respectively, i.e., $z(t)=z_{1}(t)$ for $t \geq T_{z}^{\prime}$, $\dot{e}(t)=\eta_{1}(t), \ddot{e}(t)=\eta_{2}(t)$ for $t \geq T_{e}^{\prime} . L_{z}(t), L_{e}(t)$ stand for positive continuous functions which take (based on Eqs. 13, 14 and 21) the forms $L_{z}(t)=\lambda\left(w_{B}\|v\|+w_{P}\left\|z_{1}\right\|^{2}+\right.$ $\left.w_{G}+\alpha_{0}\right)$ and $L_{e}(t)=\Lambda \frac{c}{a}\left(\mathcal{U}+c_{0}\right)+\left(w_{1}+w_{2} \| q-\right.$ $\left.q_{\text {rest }} \|\right)\left(w_{3}\|v\|\left\|\mid z_{1}\right\|+w_{4}\left\|z_{1}\right\|^{3}+w_{5}\left(\left\|z_{1}\right\|+\left\|z_{1}\right\| \alpha_{0}\right)\right)+$ $\left\|\ddot{p}_{d}\right\|$, where $\lambda \geq \lambda_{\max }\left(M^{-1}\right) ; \Lambda \geq \lambda_{\max }\left(J M^{-1} J^{T}\right)$. The quantities $L_{z}(t), L_{e}(t)$ represent physically upper estimates of the norms of $\dot{z}, \frac{d^{3} e}{d t^{3}}$ (reduced acceleration and task error jerk). Let us define concatenating control $v_{c}=$ $\left(v_{c, 1}, \ldots, v_{c, n+l-k}\right)^{T}$ as follows

$v_{c}=\left\{\begin{array}{l}v^{\prime}(t), \quad t \in\left[0, \max \left\{T_{z}^{\prime}, T_{e}^{\prime}\right\}\right], \\ v(t), \quad t \geq \max \left\{T_{z}^{\prime}, T_{e}^{\prime}\right\},\end{array}\right.$

where $v^{\prime}(t)$ is arbitrary absolutely continues mapping of time $t$ (e.g. $\left.v^{\prime}(t)=0\right) ; v(t)$ is given by Eqs. 26-27 or 36-37 or 27, (42 with $z=z_{1}, \dot{e}=\eta_{1}, \ddot{e}=\eta_{2}$ for $t \geq \max \left\{T_{z}^{\prime}, T_{e}^{\prime}\right\}$. Based on Eqs. 48-49 and 50, we are now in position to give the following theorem.

Theorem 3 If $\phi, y, e$ are only available from measurements, $J$ fulfils inequality (20) along desired trajectory $p_{d}$ and $\lambda_{0}, \lambda_{1}, \lambda_{2}, c_{0}>0, c \geq 1$ then control scheme (50) guarantees stable convergence in a finite time of the task space tracking errors $(e, \dot{e}, \ddot{e})$ to the origin $(e, \dot{e}, \ddot{e})=(0,0,0)$.

Proof The proof of Theorem 3 is a small modification of the proof of Theorem 2 from [50]. Therefore it will be omitted.

\section{Numerical Simulations}

Based on a chosen mobile manipulator task, this section demonstrates the performance of controllers proposed in the paper. The aim is to numerically compare the performances of kinematically optimal controller involving the objective function $\mathcal{F}$ with non-optimal controllers which utilise task coordinates $p_{a}$ to eliminate redundancy of the mobile manipulator. Therefore, two basic controllers given by Eqs. 26-27, $48-50$ and 36-37, 48-50 will be tested further on. For this purpose, a mobile manipulator, schematically shown in Fig. 1, is considered, where $\theta$ is the orientation angle of the platform with respect to a global coordinate system $O x_{1} x_{2} ; x_{1, c}$, $x_{2, c}$ stand for coordinates of platform centre; $\phi_{1}, \phi_{2}$ are angles of driving wheels; $y=\left(y_{1}, y_{2}\right)^{T} ; 2 W$ denotes platform width; $2 L$ is the platform length; $(a, b)$ denotes the point at which the holonomic manipulator base is fasten to the platform. In all numerical simulations, the SI units of radians, seconds, meters, etc., are used. The holonomic part (a SCARA type stationary manipulator) with two revolute kinematic pairs $(n=2)$ operating for simplicity of computations in the twodimensional task space $(m=2)$, is mounted on a platform which is assumed to be physically driven by two wheels of angular velocities $\left(\dot{\phi}_{1}, \dot{\phi}_{2}\right)^{T}$. The platform is subject to the following nonholonomic constraints $(k=3$ and $l=5)$ :

$$
\begin{aligned}
& \sin \theta \cdot \dot{x}_{1, c}-\cos \theta \cdot \dot{x}_{2, c}=0, \\
& \cos \theta \cdot \dot{x}_{1, c}+\sin \theta \cdot \dot{x}_{2, c}-R \cdot \dot{\phi}_{1}=0, \\
& \cos \theta \cdot \dot{x}_{1, c}+\sin \theta \cdot \dot{x}_{2, c}-R \cdot \dot{\phi}_{2}=0 .
\end{aligned}
$$

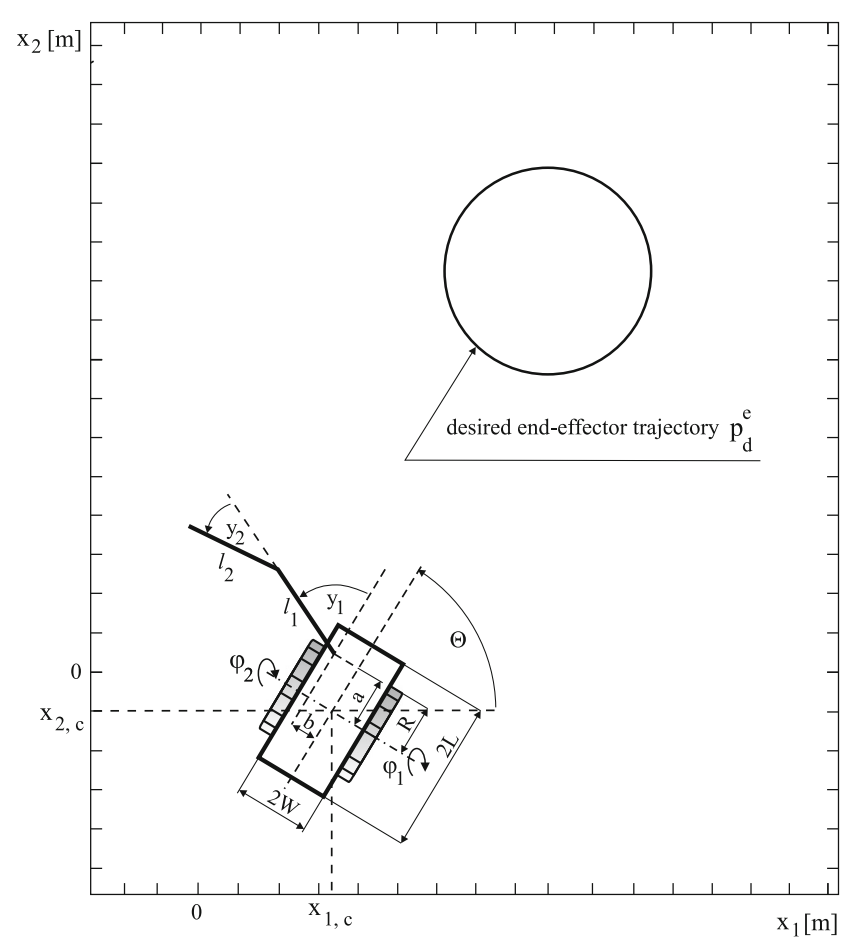

Fig. 1 A kinematic scheme of the mobile manipulator in the absolute coordinate system and the task to be accomplished 
The corresponding matrix $N(x)$ equals

$N(x)=\left[\begin{array}{cc}\cos \theta & \cos \theta \\ \sin \theta & \sin \theta \\ \frac{1}{W} & -\frac{1}{W} \\ \frac{2}{R} & 0 \\ 0 & \frac{2}{R}\end{array}\right]$,

where $x=\left(x_{1, c}, x_{2, c}, \theta, \phi_{1}, \phi_{2}\right)^{T}$. The kinematic equations of the mobile manipulator equal

$f_{e}(q)$

$=\left(\begin{array}{l}c \theta\left(a+l_{1} c y_{1}+l_{2} c y_{12}\right)-s \theta\left(b+l_{1} s y_{1}+l_{2} s y_{12}\right)+x_{1, c} \\ s \theta\left(a+l_{1} c y_{1}+l_{2} c y_{12}\right)+c \theta\left(b+l_{1} s y_{1}+l_{2} s y_{12}\right)+x_{2, c}\end{array}\right)$,

where $c \theta=\cos \theta, s \theta=\sin \theta, c y_{1}=\cos y_{1}, c y_{12}=$ $\cos \left(y_{1}+y_{2}\right), s y_{1}=\sin y_{1}, s y_{12}=\sin \left(y_{1}+y_{2}\right), l_{1}=$ $0.4=l_{2}$ stand for the link lengths of its holonomic part; $a=0.85, b=0.2 ; 2 W=0.5 ; 2 L=1.8 ; y_{1}, y_{2}$ denote joint coordinates of the holonomic manipulator. Hence, vector $q$ of the generalized coordinates takes the form $q=$ $\left(x_{1, c}, x_{2, c}, \theta, \phi_{1}, \phi_{2}, y_{1}, y_{2}\right)^{T}$. Matrix $C$ is equal to

$C=\left[\begin{array}{cc}N(x) & 0 \\ 0 & \mathbb{I}_{2}\end{array}\right]$;

reduced velocity $z$ equals $z=\left(\alpha_{1} \alpha_{2} \dot{y}_{1}, \dot{y}_{2}\right)^{T}$ and control $v=\left(v_{1}, \ldots, v_{4}\right)^{T}$, respectively. The task of the mobile manipulator is to make the end-effector follow desired circle trajectory $p_{d}^{e}=(2+\cos (t), 3+\sin (t))^{T}$ (see the solid circle in Fig. 1). On account of the fact that $l+n-m-k=$ 2 , the mobile manipulator becomes redundant mechanism. Auxiliary matrix $J(q)$ takes the following form for the mobile manipulator from Fig. 1:

$J=\left[\begin{array}{ccccccc}1 & 0 & J 13 & 0 & 0 & J 14 & J 15 \\ 0 & 1 & J 23 & 0 & 0 & J 24 & J 25 \\ -s \theta & c \theta & 0 & 0 & 0 & 0 & 0 \\ c \theta & s \theta & 0 & -R & 0 & 0 & 0 \\ c \theta & s \theta & 0 & 0 & -R & 0 & 0\end{array}\right]$

where $J_{13}=-s \theta\left(a+l_{1} c y_{1}+l_{2} c y_{12}\right)-c \theta\left(b+l_{1} s y_{1}+l_{2} s y_{12}\right)$; $J_{14}=c \theta\left(-l_{1} s y_{1}-l_{2} s y_{12}\right)-s \theta\left(l_{1} c y_{1}+l_{2} c y_{12}\right) ; J_{15}=$ $c \theta\left(-l_{2} s y_{12}\right)-s \theta l_{2} c y_{12} ; J_{23}=c \theta\left(a+l_{1} c y_{1}+l_{2} c y_{12}\right)-$ $s \theta\left(b+l_{1} s y_{1}+l_{2} s y_{12}\right) ; j 24=s \theta\left(-l_{1} s y_{1}-l_{2} s y_{12}\right)+$ $c \theta\left(l_{1} c y_{1}+l_{2} c y_{12}\right) ; J_{25}=s \theta\left(-l_{2} s y_{12}\right)+c \theta l_{2} c y_{12}$. Hence, the corresponding orthogonal complementary matrix $\mathcal{N}$ may be expressed after time-consuming but simple calculations in the form given below

$\mathcal{N}=\left[\mathcal{N}_{1}(q) \mathcal{N}_{2}(q)\right]^{T}$,

where $\mathcal{N}_{1}=\left(\mathcal{N}_{11}, \ldots, \mathcal{N}_{17}\right)^{T} ; \mathcal{N}_{2}=\left(\mathcal{N}_{21}, \ldots, \mathcal{N}_{27}\right)^{T}$; $\mathcal{N}_{11}=0 ; \mathcal{N}_{12}=0 ; \mathcal{N}_{14}=0 ; \mathcal{N}_{15}=0 ;\left(\begin{array}{c}\mathcal{N}_{13} \\ \mathcal{N}_{16} \\ \mathcal{N}_{17}\end{array}\right)=$
$\left(\begin{array}{l}J_{13} \\ J_{14} \\ J_{15}\end{array}\right) \times\left(\begin{array}{l}J_{23} \\ J_{24} \\ J_{25}\end{array}\right) ; \times$ denotes vector product; $\left(\begin{array}{l}\mathcal{N}_{21} \\ \mathcal{N}_{22}\end{array}\right)=$

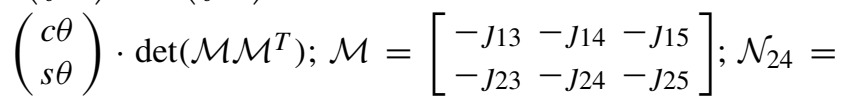
$\mathcal{N}_{25}=\frac{\operatorname{det}\left(\mathcal{M} \mathcal{M}^{T}\right)}{R} ;\left(\begin{array}{c}\mathcal{N}_{23} \\ \mathcal{N}_{26} \\ \mathcal{N}_{27}\end{array}\right)=\mathcal{M}^{T} \operatorname{adj}\left(\mathcal{M} \mathcal{M}^{T}\right)\left(\begin{array}{c}c \theta \\ s \theta\end{array}\right) ;$ $\operatorname{adj}(\cdot)$ stands for adjoint matrix of (.). Consequently, the user specified function $f_{a}(q)$ equals

$f_{a}=c_{\mathcal{F}}\left[\mathcal{N}_{1}(q) \mathcal{N}_{2}(q)\right]^{T} K_{\mathcal{F}}\left(q-q_{\text {rest }}\right)$.

The components of the nominal dynamic equations of the mobile manipulator take the following values: platform mass $m_{p}=94$; wheel mass $m_{w}=5$; platform moment of inertia $I_{p}=6.609$; the masses of the links of the holonomic manipulator equal $m_{1}=m_{2}=4$, respectively and $G(q)=0$. Initial configuration $q(0)$, reduced velocity $z(0)$ and control $v(0)$ equal $q(0)=\left(\begin{array}{lllllll}-0.4 & 0 & 0 & 0 & 0 & 0 & 0\end{array}\right)^{T}$; $z(0)=\left(\begin{array}{llll}0 & 0 & 0 & 0\end{array}\right)^{T}, v(0)=\left(\begin{array}{llll}0 & 0 & 0 & 0\end{array}\right)^{T}$, respectively in all the simulations. Moreover, $q_{\text {rest }}$ takes the form $q_{\text {rest }}=$

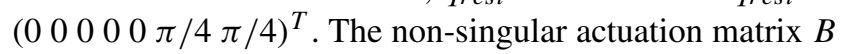
is equal to

$B=\left[\begin{array}{cccc}\frac{2}{R} & 0 & 0 & 0 \\ 0 & \frac{2}{R} & 0 & 0 \\ 0 & 0 & 1 & 0 \\ 0 & 0 & 0 & 1\end{array}\right]$

The estimates of the constants $w_{1}, \ldots, w_{6}$ which depend only on configuration $q$ can be determined based both on the numerical solution of the following system of algebraic and differential equations:

$\left(\begin{array}{c}f_{e}(q)-p_{d}^{e}(t) \\ f_{a}(q)-p_{d}^{a}(t) \\ \sin \theta \cdot \dot{x}_{1, c}-\cos \theta \cdot \dot{x}_{2, c} \\ \cos \theta \cdot \dot{x}_{1, c}+\sin \theta \cdot \dot{x}_{2, c}-R \cdot \dot{\phi}_{1} \\ \cos \theta \cdot \dot{x}_{1, c}+\sin \theta \cdot \dot{x}_{2, c}-R \cdot \dot{\phi}_{2}\end{array}\right)=0$

and the knowledge of the components of the nominal dynamic equations. Nevertheless, in order to simplify the computations, rough conservative estimates of $w_{i}, i=$ $1, \ldots, 6, a$ and $a_{e}$ have been assumed for controllers (26)(27), (48)-(50) and (36)-(37), (48)-(50). Hence, they were chosen as follows $a=a_{e}=0.1 ; w_{1}=1.5 ; w_{2}=0.001$; $w_{3}=2 ; w_{4}=3 ; w_{5}=4 ; w_{6}=0 ; \Lambda=1 ; \alpha_{0}=\alpha_{1}=0$. In order to attain the convergence of task errors $e^{e}$ less or equal to $10^{-3}$ in approximately the same time, the following numeric values of gain coefficients for both controllers are taken: $c=c_{e}=2 ; c_{0}=c_{0}^{e}=1 ; \lambda_{0}=\lambda_{0, e}=1$; $\lambda_{1}=\lambda_{1, e}=11 ; \lambda_{2}=\lambda_{2, e}=6 ; c_{\mathcal{F}}=0.01 ; K_{\mathcal{F}}=$ $\operatorname{diag}\left(\begin{array}{lllll}1 & 1 & 1 & 1 & 150\end{array}\right)$.

To speed up the convergence process of differentiators (48)-(49), we have chosen good initial guesses $z_{1}(0)$, 


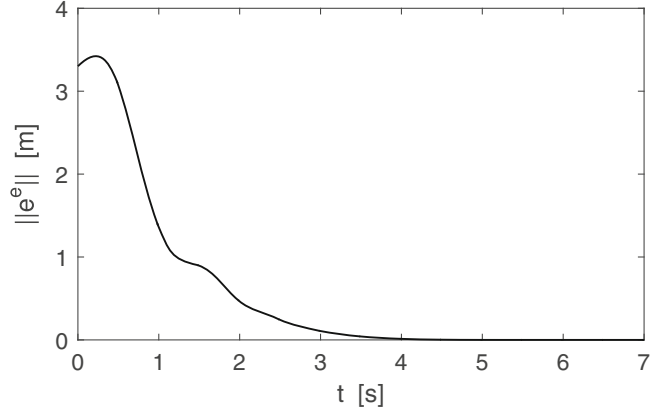

Fig. 2 Euclidean norm of task errors $e^{e}$ for controller (26)-(27), (48)-(50) with $f_{a}$ given by Eq. 38

$\eta_{0}(0), \eta_{1}(0), \eta_{2}(0)$ in the numerical examples (which imply relation $T^{\prime} \simeq \max \left\{T_{z}^{\prime}, T_{e}^{\prime}\right\} \simeq 0$ - see the proof of Theorem 3 ) based on the nominal values of our dynamic model. Consequently, differentiators (48)-(49) were run with the following initial values: $z_{1}(0)=z(0)=0, \eta_{0}(0)=$

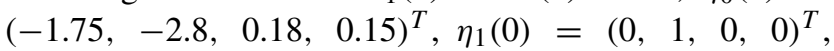
$\eta_{2}(0)=\left(\begin{array}{llll}1, & 0,0,0\end{array}\right)^{T}, v(0)=(0,0,0,0)^{T}$ and parameters $\hat{\lambda}_{0}^{e}=156 ; \hat{\lambda}_{1}^{e}=40.5 ; \hat{\lambda}_{2}^{e}=10.8 ; \hat{\lambda}_{0}^{z}=15.6$; $\hat{\lambda}_{1}^{z}=5.25$, respectively. Due to conservative nature of estimates $L_{z}$ and $L_{e}$ in Eqs. 48-49, they are assumed for simplicity of computations in all the simulations to be equal, i.e., $L_{e}(t)=L_{q}(t)$.

The first task is to track $p_{d}^{e}$ by means of non-optimal controller (26)-(27), (48)-(50) with $f_{a}=W q$, where $W=\left[\begin{array}{lllllll}0 & 0 & 0 & 0 & 0 & 1 & 0 \\ 0 & 0 & 0 & 0 & 0 & 0 & 1\end{array}\right]$ and $p_{d}^{a}(t)=(\pi / 4, \pi / 4)^{T}$ (see Remark 3.). The mobile manipulator is assumed not to be disturbed in this simulation, i.e., $D=0$. The results of the first simulation are depicted in Figs. 2, 3 and 4 which show finite-time convergence of the errors $e^{e}$ and $e^{a}$ to the origin (Figs. 2-3). Moreover, as is seen from Fig. 4, controller (26)-(27), (48)-(50) with $f_{a}$ given by Eq. 38 generates at least absolutely continuous controls $v_{c}$ with the integral norm $\overline{\left\|v_{c}\right\|}=74.5$ (defined in $L_{2}[0,7]$ norm as $\overline{\left\|v_{c}\right\|}=\sqrt{\int_{0}^{7}\left\langle v_{c}, v_{c}\right\rangle d t}$.

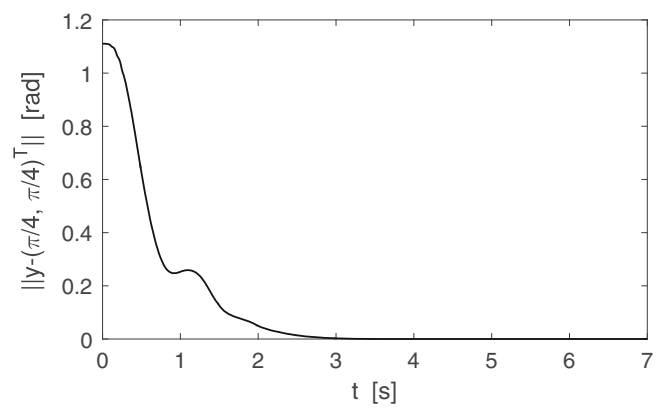

Fig. 3 Euclidean norm of task errors $e^{a}$ for controller (26)-(27), (48)-(50) with $f_{a}$ given by Eq. 38

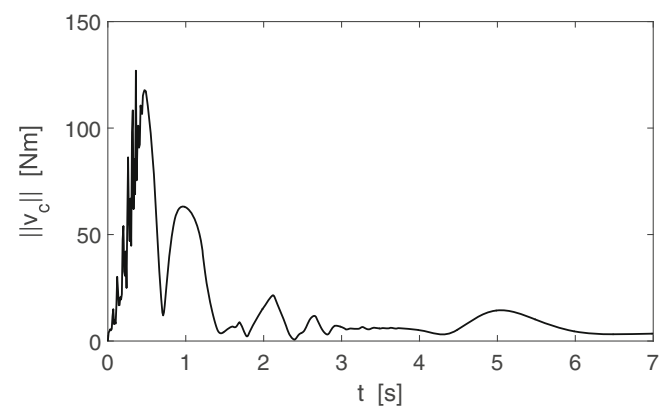

Fig. 4 Euclidean norm of controls $v_{c}$ for controller (26)-(27), (48)(50) with $f_{a}$ given by Eq. 38

In the second task, the same desired trajectory $p_{d}^{e}$ is tracked. However, controller (26)-(27), (48)-(50) involves now auxiliary function $f_{a}$ given by Eq. 57. Mobile manipulator is also not disturbed in this simulation, i.e., $\alpha_{0}=\alpha_{1}=0$. The results of the second simulation are given in Figs. 5, 6 and 7 which show finite-time convergence of $e^{e}$ and $e^{a}$ to the origin (see Figs. 5-6). From Fig. 6, it is seen, that for $t \geq 4$ controller (26)-(27), (48)-(50) generates kinematically optimal absolutely continuous torques (see Fig. 7). The integral torque norm $\overline{\left\|v_{c}\right\|}$ equals in this case $\overline{\left\|v_{c}\right\|}=46.9$ and is clearly smaller than that obtained in the first experiment.

The aim of the third simulation is to track $p_{d}^{e}$ with possibly small torque norm. For this purpose, we utilise (non-optimal) control law (36)-(37), (48)-(50) with artificially imposed velocity constraints $\dot{y}_{1}=\dot{y}_{2}=0$. Let us note that in such a case, auxiliary function $f_{a}$ does not appear in controller (36)-(37), (48)-(50). Moreover, these velocity constraints physically mean that holonomic manipulator does not move with respect to the platform. The results of the third simulation are depicted in Figs. 8 and 9. As can be seen from Fig. 8, controller (36)-(37), (48)-(50) provides finite-time accurate trajectory tracking for $t \geq 4$. Moreover, controls $v_{c}$ whose Euclidean norm is shown in Fig. 9, are at least absolutely continuous mappings. The integral torque norm $\overline{\left\|v_{c}\right\|}$ equals in this case $\overline{\left\|v_{c}\right\|}=46.05$.

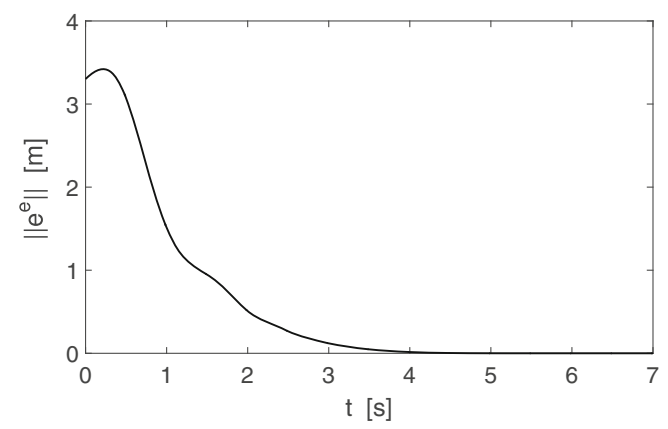

Fig. 5 Euclidean norm of task errors $e^{e}$ for kinematically optimal controller (26)-(27), (48)-(50) with $f_{a}$ given by Eq. 8 


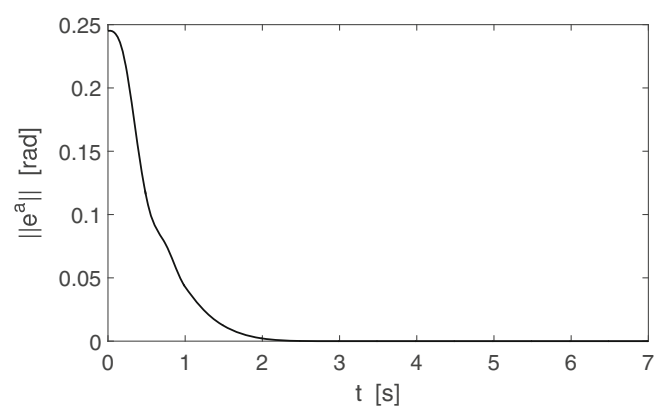

Fig. 6 Euclidean norm of task errors $e^{a}$ for kinematically optimal controller (26)-(27), (48)-(50) with $f_{a}$ given by Eq. 8

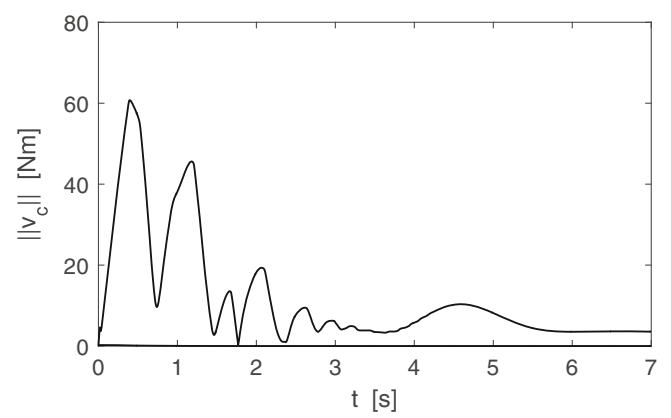

Fig. 7 Euclidean norm of steering signals $v_{c}$ for kinematically optimal controller (26)-(27), (48)-(50) with $f_{a}$ given by Eq. 8

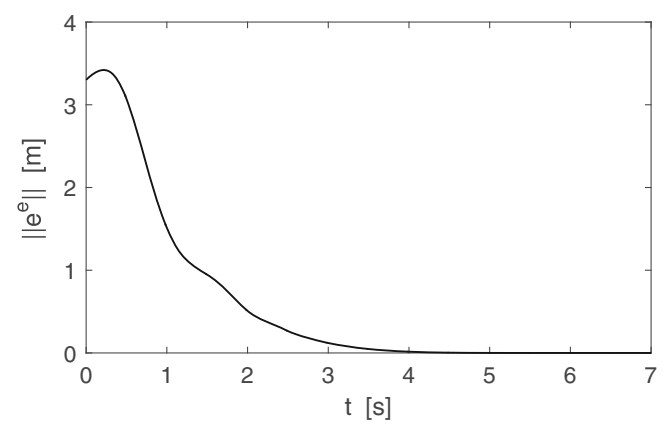

Fig. 8 Euclidean norm of task errors $e^{e}$ for non-optimal controller (36)-(37), (48)-(50)

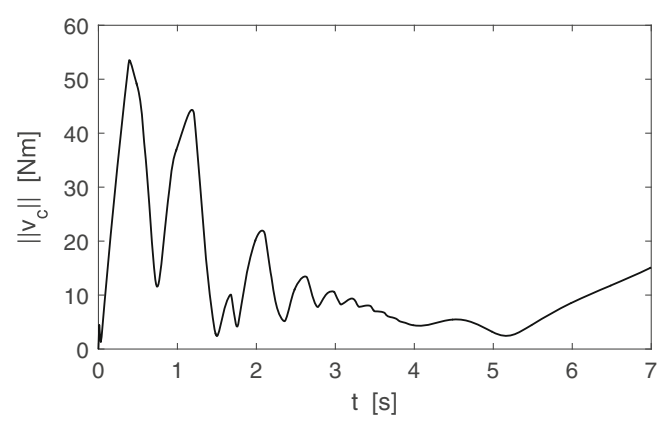

Fig. 9 Euclidean norm of steering signals $v_{c}$ for non-optimal controller (36)-(37), (48)-(50)

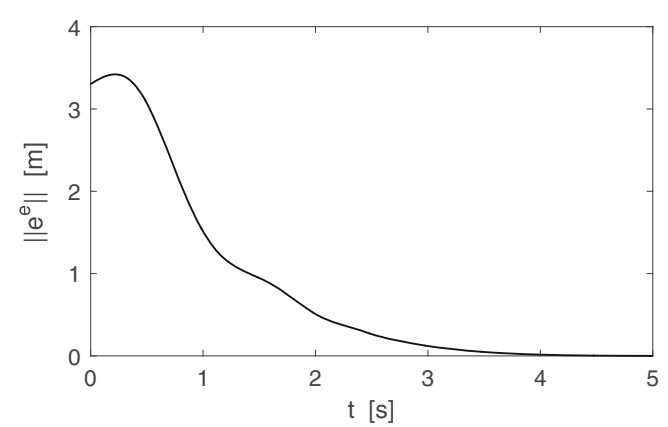

Fig. 10 Euclidean norm of task errors $e^{e}$ for kinematically optimal controller (26)-(27), (48)-(50) with both disturbance signal and measurement noise - approaching phase

Let us observe that kinematically optimal controller (26)(27), (48)-(50) generates steering signals whose integral torque norm is only slightly greater than that obtained in the current simulation.

The aim of the last (fourth) simulation is to numerically show that kinematically optimal controller (26)-(27), (48)(50) with $f_{a}$ given by (57) is robust against both disturbance signal $D \neq 0$ and a measurement noise. For this purpose, non-linear discontinuous friction term of the form $D=$ $2 z+0.5 \operatorname{sign}(z)+2 \exp \left(-0.2\|z\|^{2} \operatorname{sign}(z)\right.$ exhibiting viscous, the Coulomb and Stribeck effects [44, 45] have been added to mobile manipulator dynamic equations. Moreover, both measured angles $\phi, y$ and task error $e^{e}$ obtained from encoders, have been additionally contaminated by a measurement noise $\zeta_{i}(t)$ with two different normalised magnitudes of a Brownian motion of the form $d \zeta_{i}(t)=$ $\left\{\begin{array}{l}10^{-8} \sqrt{t} X(t) d t \text { for } t \in[0,7] \\ 10^{-6} \sqrt{t} X(t) d t \text { for } t \in(5,7]\end{array} ; X(t) \sim N(0,1), i=\right.$ $1,2,3,4,5,6$. In order to better visualise time courses of the task error and the Euclidean norm of torques, we show separately both an initial approaching phase of the end-effector to desired trajectory $p_{d}^{e}$ and the trajectory tracking phase. The results of the fourth simulation are given in Figs. 10, 11, 12, 13, 14 and 15 which indicate a good tracking performance of controller (26)-(27), (48)(50) subject to measurement noise with two different norm magnitudes (see Figs 10-13). As was expected, the measurement noise with greater normalised norm

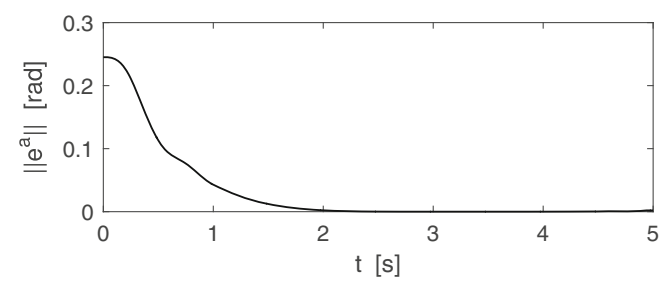

Fig. 11 Euclidean norm of task errors $e^{a}$ for kinematically optimal controller (26)-(27), (48)-(50) with both disturbance signal and measurement noise - approaching phase 


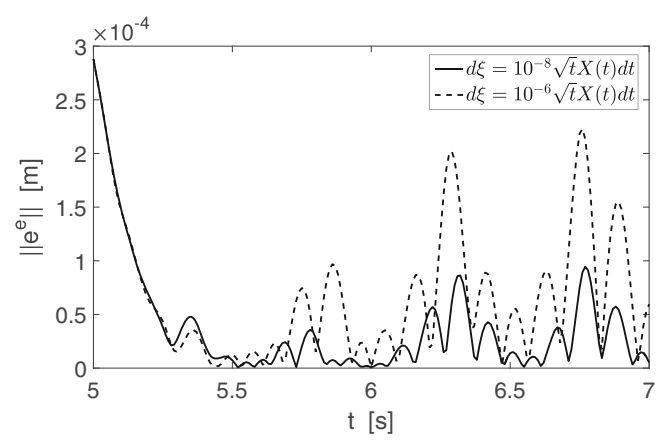

Fig. 12 Euclidean norm of task error $e^{e}$ for kinematically optimal controller (26)-(27), (48)-(50) with both disturbance signal and measurement noises - trajectory tracking phase

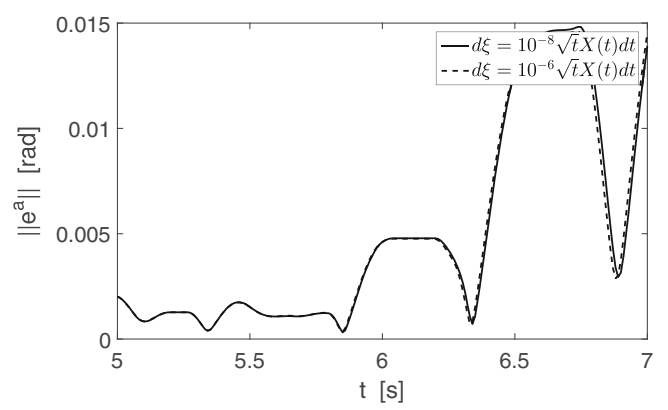

Fig. 13 Euclidean norm of task errors $e^{a}$ for kinematically optimal controller (26)-(27), (48)-(50) with both disturbance signal and measurement noises - trajectory tracking phase

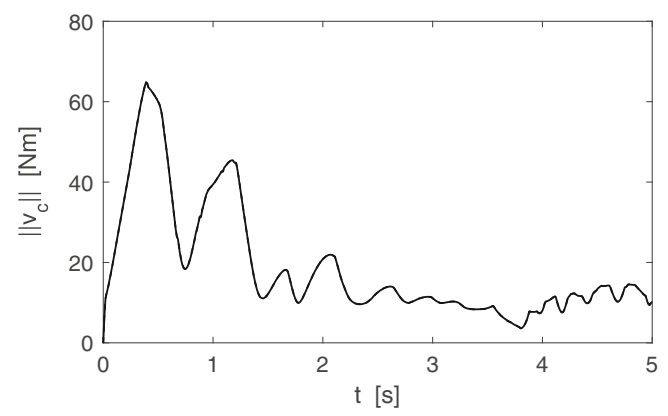

Fig. 14 Euclidean norm of torques $v_{c}$ for kinematically optimal controller (26)-(27), (48)-(50) with both disturbance signal and measurement noise - approaching phase

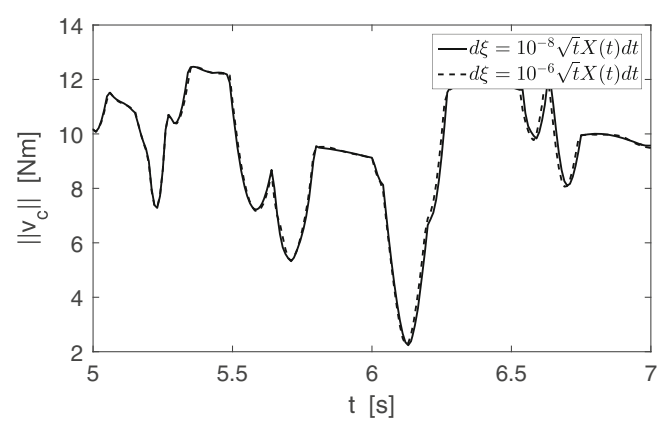

Fig. 15 Euclidean norm of steering signals $v_{c}$ for kinematically optimal controller (26)-(27), (48)-(50) with both disturbance signal and measurement noises - trajectory tracking phase magnitude (equal to $10^{-6}$ ) generates greater norms of task errors (see Figs. 12-13). The Euclidean norm of torques are depicted in Figs. 14-15. Sudden increases or decreases of $\left\|v_{c}\right\|$ in neighbourhoods of time moments 5.2, 5.7, 6.1 and 6.7 are a result of the Coulomb and Stribeck discontinuous friction term $D$ (see Fig. 15). Nevertheless, transient impetuous variations of $\left\|v_{c}\right\|$ in neighbourhood of zero reduced velocities still present absolutely continuous mappings of time.

\section{Conclusions}

The new kinematically optimal inverse-free trajectory tracking controllers have been presented in this paper. The main feature of the proposed control laws is to eliminate the inverse or pseudo-inverse of the mobile manipulator Jacobian matrix from the end-effector trajectory tracking. The Jacobian transpose matrix has been applied instead. Moreover, the presented control scheme generates absolutely continuous controls. Employing the Lyapunov stability theory, control strategies (26)-(27), (48)-(50) and (36)-(37), (48)-(50) are shown to be finite-time stable by the fulfilment of practically reasonable assumptions. One has supplied the explicit conditions on controller gains to ensure finite-time stability of the closed-loop error dynamics. Numerical simulations have made it clear that kinematically optimal controller (26)-(27), (48)-(50) with $f_{a}$ given by Eq. 8 provides steering signals of smaller $L_{2}$ norm as compared to the control scheme (26)-(27), (48)-(50) with $f_{a}$ given by Eq. 38. Although the proposed dynamically computed torque techniques need the knowledge of the system equations of the mobile manipulator, this approach is able to handle uncertainty (in dynamics and disturbance) occurring in the system. It is worth emphasizing that the absolutely continuous proposed Jacobian transpose controllers are able to cope with globally unbounded disturbances acting on the mobile manipulators as well as singular configurations that can potentially occur when tracking a desired trajectory by the end-effector. To summarize, the approach presented here may also be directly applicable to multiple mobile manipulators interacting in a six-dimensional task space.

Open Access This article is distributed under the terms of the Creative Commons Attribution 4.0 International License (http:// creativecommons.org/licenses/by/4.0/), which permits unrestricted use, distribution, and reproduction in any medium, provided you give appropriate credit to the original author(s) and the source, provide a link to the Creative Commons license, and indicate if changes were made.

Publisher's Note Springer Nature remains neutral with regard to jurisdictional claims in published maps and institutional affiliations. 


\section{References}

1. Campion, G., Bastin, G., Andrea-Novel, B.D.: Structural properties and classification of kinematic and dynamic models of wheeled mobile robots. IEEE Trans. Robot. Autom. 12(1), 47-62 (1996)

2. Yamamoto, Y., Yun, X.: Coordinating locomotion and manipulation of a mobile manipulator. IEEE Trans. Autom. Control 39(6), 1326-1332 (1994)

3. Yamamoto, Y., Yun, X.: Effect of the dynamic interaction on coordinated control of mobile manipulators. IEEE Trans. Robot. Automat. 12(5), 816-824 (1996)

4. Yamamoto, Y., Yun, X.: Unified analysis on mobility and manipulability of mobile manipulators. In: Proc. IEEE Int. Conf. Robotics and Automation, pp. 1200-1206 (1999)

5. Tzafestas, C.S., Tzafestas, S.G.: Full-state modelling, motion planning and control of mobile manipulators. Stud. Inform. Control 10(2), 109-128 (2001)

6. Mazur, A.: Trajectory tracking control in workspace-defined tasks for nonholonomic mobile manipulators. Robotica 28(2), 57-68 (2010)

7. Su, H., Krovi, V.: Decentralized dynamic control of a nonholonomic mobile manipulator collective: A simulation study. In: Proc. Dyn. Syst. and Control (2008)

8. Chung, J.H., Velinsky, S.A., Hess, R.A.: Interaction control of a redundant mobile manipulator. Int. J. Robotics Res. 17(12), 1302-1309 (1998)

9. Pajak, G., Pajak, I.: Planning of a point to point collision-free trajectory for mobile manipulators. In: Proc. 10th International Workshop on Robot Motion and Control (RoMoCo'15). Poznan, pp. 142-147 (2015)

10. Pajak, G., Pajak, I.: Point-to-point collision-free trajectory planning for mobile manipulators. J. Intell. Robot. Syst. (2016)

11. Galicki, M.: Inverse kinematics solution to mobile manipulators. Int. J. Robot. Res. 22(12), 1041-1064 (2003)

12. Boukattaya, M., Jallouli, M., Damak, T.: On trajectory tracking control for nonholonomic mobile manipulators with dynamic uncertainties and external torque forces. Robot. Auton. Syst. 60, 1640-1647 (2012)

13. Peng, J., Yu, J., wang, J.: Robust adaptive tracking control for nonholonomic mobile manipulators with uncertainties. ISA Trans. 53, 1035-1043 (2014)

14. Boukattaya, M., Damak, T., Jallouli, M.: Robust adaptive sliding mode control for mobile manipulators. Robot. Autom. Eng. 1(1), 1-6 (2017)

15. Balleieul, J.: Kinematic programming alternatives for redundant manipulators. Proc. IEEE Int. Conf. Robot. Autom. 2, 722-728 (1985)

16. De Luca, A., Oriolo, G., Giordano, P.R.: Kinematic control of nonholonomic mobile manipulators in the presence of steering wheels. In: Proc. IEEE Int. Conf. on Robotics and Automt., pp. 1792-1798 (2010)

17. Bayle, B., Fourquet, J.Y., Renaud, M.: Manipulability of wheeled mobile manipulators: Application to motion generation. Int. J. Robot. Res. 22(7-8), 565-581 (2003)

18. Fruchard, M., Morin, P., Samson, C.: A framework for the control of nonholonomic mobile manipulators. Int. J. Robot. Res. 25(8), 745-780 (2006)

19. Hammer, B., Koterba, S., Shi, J., Simmons, R., Singh, S.: An autonomous mobile manipulator for assembly tasks. Auton. Robot 28(1), 131-149 (2010)

20. Padois, V., Fourquet, J.-Y., Chiron, P.: Kinematic and dynamic model-based control of wheeled mobile manipulators: A unified framework for reactive approaches. Robotica 25(2), 1-17 (2007)
21. Galicki, M.: Task space control of mobile manipulators. Robotica 29(2), 221-232 (2010)

22. Galicki, M.: Collision-free control of mobile manipulators in a task space. Mech. Syst. Signal Process. 25(7), 2766-2784 (2011)

23. Galicki, M.: Two-stage constrained control of mobile manipulators. Mech. Mach. Theory 54, 18-40 (2012)

24. Galicki, M.: Real-time constrained trajectory generation of mobile manipulators. Robot. Auton. Syst. 78, 49-62 (2016)

25. Andaluz, V., Robeti, F., Toibero, J.M., Carelli, R.: Adaptive unified motion control of mobile manipulators. Control. Eng. Pract. 20, 1337-1352 (2012)

26. Shamir, T., Yomdin, Y.: Repeatability of redundant manipulators: Mathematical solution of the problem. IEEE Trans. Automat. Cont. 33(11), 1004-1009 (1988)

27. Roberts, R.G., Maciejewski, A.A.: Nearest optimal repeatable control strategies for kinematically redundant manipulators. IEEE Trans. Robot. Automat. 8(3), 327-337 (1992)

28. Nakamura, Y., Hanafusa, H.: Inverse kinematic solutions with singularity robustness for robot manipulator control. J. Dyn. Syst. Meas. Control 108(3), 163-171 (1986)

29. Wampler, C.W., Leifer, L.J.: Applications of damped least-squares methods to resolved-rate and resolved-acceleration control of manipulators. J. Dyn. Syst. Meas. Control 110(1), 31-38 (1988)

30. Shevitz, D., Paden, B.: Lyapunov stability theory of nonsmooth systems. IEEE Trans. Automat. Contr. 30(9), 1910-1914 (1994)

31. Tanner, H.G., Kyriakopoulos, K.J.: Nonholonomic motion planning for mobile manipulators. In: Proc. Int. Conf. Robotics and Automation, vol. 2, pp. 1233-1238 (2000)

32. Tanner, H.G., Loizou, S.G., Kyriakopoulos, K.J.: Nonholonomic navigation and control of cooperating mobile manipulators. IEEE Trans. Rob. and Aut. 19(1), 53-64 (2003)

33. Spong, M.W., Vidyasagar, M.: Robot Dynamics and Control. Wiley, New York (1989)

34. Siciliano, B., Sciavicco, L., Villani, L., Oriolo, G.: Robotics: Modelling, Planning and Control. Springer Verlag, London (2010)

35. Galicki, M.: Constraint finite-time control of redundant manipulators. Int. J Robust Nonlinear Control 27(4), 639-660 (2016a)

36. Galicki, M.: Finite-time-trajectory tracking control in a task space of robotic manipulators. Automatica 67, 165-170 (2016)

37. Galicki, M.: Optimal kinematic finite-time control of mobile manipulators. In: Proc. RoMoCo 2017 IEEE Explore, pp. 129-134 (2017)

38. Feng, Y., Yu, X., Man, Z.: Non-singular terminal sliding mode control of rigid manipulators. Automatica 38(12), 2159-2167 (2002)

39. Yu, S., Yu, X., Shirinzadeh, B., Man, Z.: Continuous finitetime control for robotic manipulators with terminal sliding mode. Automatica 41, 1957-1964 (2005)

40. Zhao, D., Li, S., Gao, F.: A new terminal sliding mode control for robotic manipulators. Int. J. Control. 82(10), 1804-1813 (2009)

41. Filippov, A.F.: Differential Equations with Discontinuous Righthand Side. Kluwer, Dordrecht (1988)

42. Levant, A.: Higher-order sliding modes, differentiation and output-feedback control. Int. J. Control. 76(9-10), 924-941 (2003)

43. Levant, A., Livne, M.: Exact differentiation of signals with unbounded higher derivatives. IEEE Trans. Autom. Control 57(4), 1076-1080 (2012)

44. Haessing, D., Friedland, B.: On the modeling and simulation of friction. Trans. ASME J. Dyn. Syst. Meas. Control 113(3), 354-362 (1991)

45. Canudas de Wit, C., Ollson, H., Astrom, K., Lischinsky, P.: A new model for control of systems with friction. IEEE Trans. Automat. Contr. 40(3), 419-425 (1995) 
46. Zadarnowska, K., Tchon, K.: A control theory framework for performance evaluation of mobile robots. Robotica 26(6), 703715 (2007)

47. Tchon, K.: On kinematic singularities of nonholonomic robotic systems. Proc. RoManSy 13, 75-84 (2000)

48. Li, Z., Ge, S.S.: Fundamentals of Modeling and Control of Mobile Manipulators. CRC (2013)

49. Tchon, K.: Repeatability of inverse kinematics algorithms for mobile manipulators. IEEE Trans. Automat. Control 47(8), 13761380 (2002)

50. Galicki, M.: Robust task space finite-time chattering-free control of robotic manipulators. J. Intell. Robot. Syst. 85, 471-489 (2017)

51. Hong, Y.G.: Finite-time stabilization and stabilizability of a class of controllable systems. Syst. Control Lett. 46(4), 231-236 (2002)

52. Gauthier, J.-P., Monroy-Perez, F.: On certain hyperelliptic signals that are natural controls for nonholonomic motion planning. Math. Control signals Syst. 27, 415-437 (2015)

53. Zuyev, A., Grushkovskaya, V.: Motion planning for control-affine systems satisfying low-order controllability conditions. Int. J. Control, 1-29 (2017)
54. Minguez, J., Lamiraux, F., Laumond, J.P.: Motion planning and obstacle avoidance. In: Springer Handbook of Robotics, pp. 11771201 (2016)

Mirosław Galicki received the M. Sc. degree from the Technical University of Wroclaw, Poland, in 1980 and the Ph. D. degree in electrical and computer sciences from the Technical University of Wroclaw. He was with the Institute of Technical Cybernetics of Wroclaw from September 1981-1984. Since 1985 he has been at the University of Zielona Gora, Poland, and from 1992-2013 he was also with the Institute of Medical Statistics, Computer Sciences and Documentation of the Friedrich Schiller University Jena, Germany. His research interests include control of dynamic systems with both holonomic and non-holonomic constraints, structural optimization and optimal control problems. Areas of particular interest include the problems of motion control of both redundant stationary and mobile manipulators. 